\title{
Generalized models for high-throughput analysis of uncertain nonlinear systems
}

\author{
Thilo Gross • Stefan Siegmund
}

Accepted: 12 December 2011 / Published online: 12 December 2011

(C) 2011 Gross, Siegmund; licensee Springer. This is an Open Access article distributed under the terms of the Creative Commons Attribution License (http://creativecommons.org/licenses/by/2.0), which permits unrestricted use, distribution, and reproduction in any medium, provided the original work is properly cited.

\begin{abstract}
Purpose: Describe a high-throughput method for the analysis of uncertain models, e.g. in biological research.

Methods: Generalized modeling for conceptual analysis of large classes of models. Results: Local dynamics of uncertain networks are revealed as a function of intuitive parameters.

Conclusions: Generalized modeling easily scales to very large networks.
\end{abstract}

Keywords Generalized modeling · high-throughput method · uncertain models · biological research

\section{Background}

The ongoing revolution in systems biology is revealing the structure of important systems. For understanding the functioning and failure of these systems, mathematical modeling is instrumental, cp. Table 1 . However, application of the traditional modeling paradigm, based on systems of specific equations, faces some principal difficulties in these systems. Insights from modeling are most desirable during the early stages of exploration of a system, so that insights from modeling can feed into experimental set ups.

\footnotetext{
T Gross

Max-Planck Institute for the Physics of Complex Systems, Nöthnitzer Str. 38, 01187 Dresden, Germany

e-mail: thilo.gross@physics.org

T Gross $\cdot$ S Siegmund $(\bowtie)$

Department of Mathematics, Center for Dynamics, Technical University Dresden, 01062 Dresden, Germany

e-mail: siegmund@tu-dresden.de

T Gross

e-mail: gross@pks.mpg.de
} 
Table 1 Three different levels of modeling.

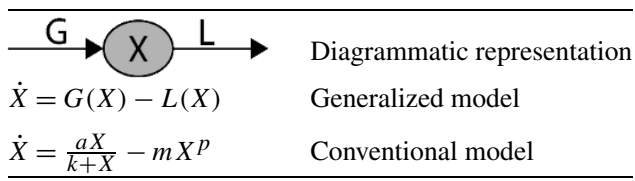

However, at this stage the knowledge of the system is often insufficient to restrict the processes to specific functional forms. Further, the number of variables in the current models prohibits analytical investigation, whereas simulation does not allow efficient exploration of large parameter spaces.

\section{Method}

Here we present the approach of generalized modeling. The idea of this approach is to consider not a single model but the whole class of models which are plausible given the available information. Modeling can start from a diagrammatic sketch, which is translated into a generalized model containing unspecified functions. Although such models cannot be studied by simulation, other tools can be applied more easily and efficiently than in conventional models. In particular, generalized models reveal the dynamics close to every possible steady state in the whole class of systems depending on a number of parameters that are identified in the modeling process.

\section{Results}

In the past it has been shown that generalized modeling enables high-throughput analysis of complex nonlinear systems in various applications [1, 2]. In particular it was shown that generalized models can be used to obtain statistically highly-significant results on systems with thousands of unknown parameters [3].

\section{Discussion}

For illustration consider a population $X$ subject to a gains $G$ and losses $L$,

$$
\frac{d}{d t} X=G(X)-L(X)
$$

where $G(X)$ and $L(X)$ are unspecified functions. We consider all positive steady states in the whole class of systems described by Equation 1 and ask which of those states are stable equilibria. For this purpose denote an arbitrary positive steady state of the system by $X^{*}$, i.e. $X^{*}$ is a placeholder for every positive steady state that exists in the class of systems. For determining the stability of $X^{*}$ one can use dynamical systems theory and evaluate the Jacobian of Equation 1 at $X^{*}$

$$
\mathbf{J}^{*}=\left.\frac{\partial G}{\partial X}\right|_{X=X^{*}}-\left.\frac{\partial L}{\partial X}\right|_{X=X^{*}} .
$$


For expressing the Jacobian as a function of easily interpretable parameters we use the identity $\left.\frac{\partial F}{\partial X}\right|_{X=X^{*}}=\left.\frac{F\left(X^{*}\right)}{X^{*}} \frac{\partial \log F}{\partial \log X}\right|_{X=X^{*}}$, which holds for positive $X^{*}$ and $F\left(X^{*}\right)$. We write

$$
\mathbf{J}^{*}=\frac{G\left(X^{*}\right)}{X^{*}} g_{X}-\frac{L\left(X^{*}\right)}{X^{*}} \ell_{X},
$$

where $g_{X}:=\left.\frac{\partial \log G}{\partial \log X}\right|_{X=X^{*}}$ and $\ell_{X}:=\left.\frac{\partial \log L}{\partial \log X}\right|_{X=X^{*}}$ are so-called elasticities, a term mainly used in economics. The prefactors $\frac{G\left(X^{*}\right)}{X^{*}}$ and $\frac{L\left(X^{*}\right)}{X^{*}}$ denote per-capita gain and loss rates, respectively. By Equation 1 gain and loss rates balance in the steady state $X^{*}$ such that we can define

$$
\alpha:=\frac{G\left(X^{*}\right)}{X^{*}}=\frac{L\left(X^{*}\right)}{X^{*}},
$$

which can be interpreted as a characteristic turnover rate of $X$. We can thus write the Jacobian at $X^{*}$ as

$$
\mathbf{J}^{*}=\alpha\left(g_{X}-\ell_{X}\right) .
$$

To interpret $g_{X}$ and $\ell_{X}$ note that for any power law $L(X)=m X^{p}$ the elasticity is $\ell_{X}=p$. Constant functions have an elasticity 0 , all linear functions an elasticity 1 , quadratic functions an elasticity 2. This also extends to decreasing functions, e.g. $G(X)=\frac{m}{X}$ has elasticity $g_{X}=-1$. For more complex functions $G$ and $L$ the elasticities can depend on the location of the steady state $X^{*}$. However, even in this case the interpretation of the elasticity is intuitive, e.g. the Holling type-II functional response $G(X)=\frac{a X}{k+X}$ is linear for low density $X\left(g_{X} \approx 1\right)$ and saturates for high density $X$ $\left(g_{X} \approx 0\right)$.

So far we succeeded in expressing the Jacobian of the model as a function of three easily interpretable parameters. A steady state $X^{*}$ in a dynamical system is stable if and only if the real parts of all eigenvalues of the Jacobian are negative. In the present model this implies that a given steady state is stable whenever the elasticity of the loss exceeds the elasticity of the gain $g_{X}<\ell_{X}$. A change of stability occurs if $g_{X}=\ell_{X}$ as Equation 1 undergoes a saddle-node bifurcation.

\section{Conclusion}

The simple example already shows that generalized modeling

- reveals boundaries of stability, valid for a class of models and robust against uncertainties in specific models

- avoids expensive numerical approximation of steady states and can be scaled to high-dimensional models

Also in larger models it is generally straight forward to derive an analytical expression that states the Jacobian of the generalized model as a function of simple parameters. This Jacobian can then analyzed analytically or numerically by a random sampling procedure. Both approaches are illustrated in a recent paper on bone remodeling [4]. Here, the generalized model analysis showed that the area of parameter space most likely realized in vivo is close to Hopf and saddle-node bifurcations, which enhances 
responsiveness, but decreases stability against perturbations. A system operating in this parameter regime may therefore be destabilized by small variations in certain parameters. Although theoretical analysis alone cannot prove that such transitions are the cause of pathologies in patients, it is apparent that a bifurcation happening in vivo would lead to pathological dynamics. In particular, a Hopf bifurcation could lead to oscillatory rates of remodeling that are observed in Paget's disease of bone. This result illustrates the ability of generalized models to reveal insights into systems on which only limited information is available.

\section{Competing interests}

The authors declare that they have no competing interests.

\section{Authors' contributions}

The authors have developed this note jointly. The method of generalized modeling was invented by the first author.

\section{References}

1. Gross, T., Feudel, U.: Generalized models as a universal approach to the analysis of nonlinear dynamical systems. Phys. Rev. E 73, 016205 (2006)

2. Steuer, R., Gross, T., Selbig, J., Blasius, B.: Structural kinetic modeling of metabolic networks. PNAS 103, 11868 (2006)

3. Gross, T., Rudolf, L., Levin, S.A., Dieckmann, U.: Generalized models reveal stabilizing factors in food webs. Science 320, 747 (2009)

4. Zumsande, M., Stiefs, D., Siegmund, S., Gross, T.: General analysis of mathematical models for bone remodeling. Bone (2011). doi:10.1016/j.bone.2010.12.010 